\title{
A theory free from loopholes?
}

If quantum theory is correct, then the world is fundamentally different from what most of us suppose. That's the gist of John Bell's famous 'theorem' of forty-one years ago, which suggests that quantum theory is inconsistent with so-called local realism - the notion that the world exists 'out there' with properties of its own, and that physical influences always act locally, without any 'spooky' action at a distance.

Quantum theory could be wrong, of course, but many experiments back it up - and I don't know a single physicist who finds this surprising. Measure the properties of pairs of entangled particles such as the polarization of photons sent to very distant locations - and you find correlations stronger than any locally realistic picture can explain. Every few months another experiment gives similar confirmation, with better statistics, and you have to wonder: do further experiments have any point? It is tempting to answer no, but the issues involved could be more subtle than many physicists suppose.

It is widely acknowledged that no experiment has yet been truly 'loophole free'. In particular, photon detectors only detect a fraction of the photons in any experiment. If these pairs give an unbiased sample of all pairs, this is no problem. But what if the physics that determines which photons get detected is somehow linked to the physics that underlies the correlations?

That may seem unlikely, and closing this detection loophole may be no more than a moppingup operation. "It is hard for me to believe," Bell himself commented, "that quantum mechanics works so nicely for inefficient practical setups and is yet going to fail badly when sufficient refinements have made." Then again, we cannot know the results of an experiment in advance.

In 1933, sensible physicists thought Otto Stern was wasting his time measuring the magnetic moment of the proton, as this particle would obviously behave much as the electron, following Dirac's theory. But Stern found a moment two-and-a-half times larger than expected - prefiguring the discovery of internal nucleon structure. It makes sense to close loopholes, whatever our expectations.

Besides, there's another possibility that Bell, apparently, did not consider - that local realism might still prevail, even if quantum

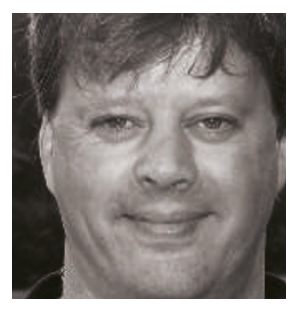

theory doesn't fail. Ian Percival, among others, has argued that even if quantum theory is strictly correct, it could be that some quantum states - in particular those that allow 'weak non-locality' of the kind that violates local realism - might be impossible to realize in practice. The analogy would be with the

I HAVE TO

ADMIT TO A

SNEAKING HOPE

THAT SOME NEW

PHYSICS WILL

EMERGE THAT

MAKES OUR

WORLD LOCALLY

REALISTIC

AFTER ALL.

\section{Return to the space race}

The future of space science and exploration has been much discussed over the past few months, on both sides of the Atlantic. In September, NASA administrator Michael Griffin announced plans for returning humans to the Moon, as a stepping stone to Mars and beyond. It would supposedly cost US\$104 billion, would stay within NASA's existing budget and would have astronauts on the Moon by 2018 .

A month later, the UK Royal Astronomical Society issued a report on the "Scientific Case for Human Space Flight", arguing that Britain should take part in the new space race. Among the claimed benefits was that human space exploration motivates children to get interested in science.

There's no denying that. Shar- ing a stage with an astronaut (as I have) is like standing beside a rock star. There is also no denying that returning to the Moon has greater potential scientific benefit than continuing the space shuttle and the International Space Station. But then it is hard to think of any US\$100-billion project that would not have greater scientific benefit.

If it's a question of inspiring young people to learn science, it would be cheaper and, perhaps, wiser to follow the suggestion of a recent National Academy of Science report, which proposed spending money to train teachers in public schools to teach science, and to teach it creatively.

There is cause for concern that the NASA plan will hurt science, even if it does get us back to the

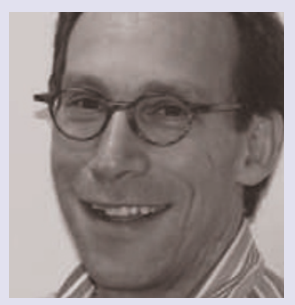

I AM ALL FOR ADVENTURE, BUT BE HONEST ABOUT THE COSTS AND THE BENEFITS FOR SCIENCE.
Moon (which is also debatable). Working within the current budget implies the reallocation of resources from other NASA programmes. If history is any guide, those programmes are likely to be the ones that involve real science, and which up to now have used much cheaper unmanned probes such as the Mars Rovers. Exciting projects - such as the search for gravitational waves, new probes of the cosmic microwave background, and the successor to the Hubble space telescope - have already been delayed by the shifting of funds from unmanned to manned space exploration. I am all for adventure, but only if we are honest about the costs and the benefits for science as well. Lawrence M. Krauss 